\title{
Video Article \\ Microinjection-based System for In Vivo Implantation of Embryonic Cardiomyocytes in the Avian Embryo
}

\author{
Trevor Henley ${ }^{1}$, Kandace Thomas ${ }^{1}$, Michael Bressan ${ }^{1}$ \\ ${ }^{1}$ Department of Cell Biology and Physiology, McAllister Heart Institute, University of North Carolina at Chapel Hill
}

Correspondence to: Michael Bressan at michael_bressan@med.unc.edu

URL: https://www.jove.com/video/59267

DOI: doi:10.3791/59267

Keywords: Developmental Biology, Issue 144, embryonic development, in vivo, implantation, cellular integration, cell tracing, cardiac lineage Date Published: 2/17/2019

Citation: Henley, T., Thomas, K., Bressan, M. Microinjection-based System for In Vivo Implantation of Embryonic Cardiomyocytes in the Avian Embryo. J. Vis. Exp. (144), e59267, doi:10.3791/59267 (2019).

\section{Abstract}

Interpreting the relative impact of cell autonomous patterning versus extrinsic microenvironmental influence on cell lineage determination represents a general challenge in developmental biology research. In the embryonic heart, this can be particularly difficult as regional differences in the expression of transcriptional regulators, paracrine/juxtacrine signaling cues, and hemodynamic force are all known to influence cardiomyocyte maturation. A simplified method to alter a developing cardiomyocyte's molecular and biomechanical microenvironment would, therefore, serve as a powerful technique to examine how local conditions influence cell fate and function. To address this, we have optimized a method to physically transplant juvenile cardiomyocytes into ectopic locations in the heart or the surrounding embryonic tissue. This allows us to examine how microenvironmental conditions influence cardiomyocyte fate transitions at single cell resolution within the intact embryo. Here, we describe a protocol in which embryonic myocytes can be isolated from a variety of cardiac sub-domains, dissociated, fluorescently labeled, and microinjected into host embryos with high precision. Cells can then be directly analyzed in situ using a variety of imaging and histological techniques. This protocol is a powerful alternative to traditional grafting experiments that can be prohibitively difficult in a moving tissue such as the heart. The general outline of this method can also be adapted to a variety of donor tissues and host environments, and its ease of use, low cost, and speed make it a potentially useful application for a variety of developmental studies.

\section{Video Link}

The video component of this article can be found at https://www.jove.com/video/59267/

\section{Introduction}

Cardiac developmental research has benefitted enormously from the advent of germline transgenic model systems which have identified many of the gene regulatory networks that pattern different cell lineages and functional domains in the heart. However, identifying how these gene networks interact with and respond to microenvironmental conditions, including paracrine/juxtacrine signals and biophysical inputs (stretch, strain, hemodynamic flow), can be challenging. As such, it is not always easy to determine whether a cellular phenotype arises as a direct consequence of a genetic perturbation or as a secondary result of an adaption to changes in cardiac biomechanics or higher order tissue composition $^{1,2}$

Grafting experiments, which have classically been used to address concepts of fate specification, commitment, induction, and competence ${ }^{3,4}$, would represent an ideal approach to circumvent some of the challenges inherent in defining cell autonomous versus environmental influence in the heart. Unfortunately, heart contractions make standard grafting approaches difficult. Rapid movement of the tissue often prevents grafted cells from adhering to the heart and large tissue punctures (normally required for grafting) frequently lead to heart failure and embryonic lethality ${ }^{5,6,7}$. Therefore, we have developed a pressure-based, microinjection system for precision cellular implantation into the developing chick heart, circumventing the technical hurdles of tissue grafting described previously ${ }^{8,9}$. Using this technique, individual or small groups of cardiac cells isolated from a donor embryo can be microinjected into a variety of regions of a host embryonic heart eliminating the need for extensive host preparation and the large tissue insults that arise using standard grafting techniques. The microinjection needles used for these implantation studies have an outer diameter of $\sim 30-40 \mu \mathrm{m}$, which means that the needle can be placed directly in the target tissue (i.e., can penetrate the embryonic myocardial wall) and cells can be focally delivered with minimal damage to the surrounding tissue. The protocol can be used to perform a variety of isotopic, heterotopic, isochoric, and heterochronic manipulations, providing a rapid, flexible, and low-cost approach to directly examine classical experimental embryological paradigms in the developing four-chambered heart.

In the protocol outlined below, we label donor cells with a cell permeant fluorescent dye, which allows for the success of a microinjection experiment to be monitored in real-time and the location of engrafted cells to be documented without the need for any additional staining. However, it should be noted that this approach is best suited for short term experiments (approximately $48 \mathrm{~h}$ ) as the fluorescent dye can be lost through cell division. Alternative approaches can be used for longer term experiments. 
While we are presenting this technique in the context of cardiac development, we have used it to great effect for cell implantation experiments into the mesoderm, head, limbs, and somites. As such the basic approach described below is highly tractable and can be used in a variety of organ systems.

\section{Protocol}

All methods described adhere to animal care guidelines of The University of North Carolina at Chapel Hill.

\section{Preparation of micro-injection pipettes}

1. Pull glass capillaries using a micropipette puller. For some injections, needle beveling is recommended as it provides an extremely sharp surface devoid of structural impurities. To do this, polish the end of a pulled needle on a beveling wheel at an angle of $45^{\circ}$ for $15-20$ min. NOTE: Exact settings for pulling will vary based on the puller being used. The final inner diameter of the bevel should be between $20-40 \mu \mathrm{m}$.

2. Coat the inner and external surfaces of the glass capillary with silicone. First dip the needles in the siliconizing agent to coat the external surface of the needle and then backload the siliconizing agent solution into each micropipette to coat the inner surface.

NOTE: Coating of the glass capillaries should be done $24 \mathrm{~h}$ before the implantation experiment. Coating the glass capillaries with silicone provides a chemically inert surface to the glass. If the capillaries are left untreated, the cell suspension generated in later steps will adhere to the glass and plug the needle. Therefore, coating is necessary and vital to the success of the method.

CAUTION: The siliconizing agent is a ready-made commercially available mixture of heptane and 1,7-dichloro-1,1,3,3,5,5,7,7octamethyltetrasiloxane (Table of Materials). It is extremely flammable and acutely toxic. Always handle with proper PPE inside a fume hood.

1. To backload the needle, load $\sim 5-10 \mu \mathrm{L}$ of the siliconizing agent into a micro-injection pipette tip (Table of Materials). Place the microinjection tip in the wide end of the pulled glass capillary and position the tip as far down as possible (close to the glass needle tip). Eject the siliconizing agent while slowly removing the loading pipette in order to minimize air bubbles in the needle.

2. Leave the siliconizing agent in the glass needle for $10 \mathrm{~min}$, remove by aspirating with a new loading pipette and allow needles dry overnight in a fume hood.

3. The morning of the experiment, rinse the glass capillaries with deionized water following the procedure in step 1.2 and allow to dry for $3-4 \mathrm{~h}$.

\section{Preparation of solutions}

1. Prepare $5 \mathrm{~mL}$ of trypsin neutralizing solution by supplementing $4.2 \mathrm{~mL}$ of Dulbecco's modified Eagle's medium and Ham's nutrient mixture F12 (DMEM/F12) with $750 \mu \mathrm{L}$ of Fetal Bovine Serum (FBS) and $50 \mu \mathrm{L}$ of Penicillin/Streptomycin. Store at $37^{\circ} \mathrm{C}$ until use.

2. Prepare $5 \mu \mathrm{M}$ labeling dye solution by pipetting $5 \mu \mathrm{L}$ of $1 \mathrm{mM}$ stock labeling dye (in dimethyl sulfoxide [DMSO], Table of Materials) into 995 $\mu \mathrm{L}$ of Hank's balanced salt solution (HBSS). Vortex for $1 \mathrm{~min}$ and store at $37^{\circ} \mathrm{C}$ until use.

3. Prepare fresh paraformaldehyde (PFA) by combining $10 \mathrm{~mL}$ of $32 \%$ PFA stock solution with $62 \mathrm{~mL}$ of molecular biology grade water and 8 $\mathrm{mL}$ of $10 x$ Dulbecco's phosphate-buffered saline (DPBS). The final concentration is 4\% PFA in 1x DPBS.

\section{Preparation of host embryos}

1. Incubate fertile chicken eggs in a horizontal orientation in a humidified incubator at $38^{\circ} \mathrm{C}$ until Hamburger and Hamilton $(\mathrm{HH}) \mathrm{Stage} 19^{10}$. NOTE: The stage chosen for manipulation is flexible and entirely dependent upon the aims of each individual experiment.

2. Score the "flat" end of the egg shell along the egg equator using angled forceps to make a small puncture $>1 \mathrm{~mm}$ in diameter. Insert an $18 \mathrm{G}$ needle with attached $10 \mathrm{~mL}$ syringe through the puncture and remove $\sim 5 \mathrm{~mL}$ of albumin.

NOTE: This anatomical location is external to the "air cell" inside the egg and keeps albumin from leaking once the puncture is made. This step is recommended as it "drops" the embryo away from the egg shell, preventing potential damage in the subsequent steps.

3. Apply transparent tape to the top of the egg shell. Score with angled forceps and cut a $\sim 2.5 \mathrm{~cm}$ window using curved tenotomy scissors.

4. Inspect and stage the embryo based on criteria established by Hamburger and Hamilton ${ }^{10}$ and seal the puncture from step 3.2 with transparent tape.

NOTE: Here, stage HH 19 embryos are used which have 37-40 somites extending into the tail bud. The puncture should not be sealed until after the window is opened along the top of the egg (step 3.3).

5. Inject $\sim 200 \mu \mathrm{L}$ of India Ink/HBSS mixture (1:5) beneath the embryo using a $32 \mathrm{G}$ needle with attached $1 \mathrm{~mL}$ syringe. NOTE: India ink provides visual contrast between the embryo and the yolk beneath. Alternative dyes such as neutral red or commercially available cyan fluorescent protein (CFP) or blue fluorescent protein (BFP) fluorescence filter sets can be used to improve contrast.

6. Add $1 \mathrm{~mL}$ of HBSS dropwise onto the embryonic disc and seal windowed shells with paraffin film. Place eggs back in the humidified incubator until ready for injection.

\section{Isolation of donor tissue}

1. Incubate donor fertile chicken eggs in a humidified incubator at $38{ }^{\circ} \mathrm{C}$ until Stage $\mathrm{HH} 19$ (or desired stage).

2. Remove the embryo from the egg and place in a $100 \mathrm{~mm} \times 15 \mathrm{~mm}$ petri dish containing sterile HBSS at room temperature (RT).

3. Surgically microdissect atrial donor tissue from each embryo by first isolating the entire embryonic heart from the embryo and then by isolating the atria from the heart using forceps, tenotomy scissors, and microspatula under a stereo dissecting microscope. Pool in a sterile $1.5 \mathrm{~mL}$ microcentrifuge tube containing $1 \mathrm{~mL}$ of HBSS on ice.

4. Once all donor tissue has been collected, pellet the tissue by centrifugation at $1000 \times g$ for 5 min at $4{ }^{\circ} \mathrm{C}$ in a fixed-angle microcentrifuge. 


\section{Trypsin digestion of donor tissue}

1. Resuspend cell pellets in $1 \mathrm{~mL}$ of prewarmed $0.05 \%$ trypsin-EDTA and incubate at $37^{\circ} \mathrm{C}$ for $15 \mathrm{~min}$ in a shaking heat block at $300 \mathrm{rpm}$. Alternatively, use a water bath with periodical agitation of the sample.

2. Pipette the digestion solution up and down to break up any remaining tissue, and pellet as in step 4.4 .

3. Resuspend the pellet in $1 \mathrm{~mL}$ of the trypsin neutralizing solution and centrifuge as in step 4.4 .

4. Resuspend the cells in $400 \mu \mathrm{L}$ of red fluorescent labeling dye solution and incubate at $37^{\circ} \mathrm{C}$ for 20 min in a heat block. Alternatively, use a water bath.

5. Once the labeling reaction is finished, pellet the cells as in step 4.4 and wash with $1 \mathrm{~mL}$ of HBSS (number of wash steps can be varied between 1 and 3).

6. Resuspend the labeled, pelleted cells at a concentration of $\sim 50,000$ cells $/ \mu \mathrm{L}$, which generally results in a $5-10 \mu \mathrm{L}$ working volume depending on the total cell yield. NOTE: Cell concentrations below $\sim 50,000$ cells $/ \mu \mathrm{L}$ can result in poor injection efficiency.

\section{In-vivo injection}

1. Backload the cell suspension into a silicone treated glass capillary pipette following the procedures in step 1.2. Mount the pipette into the pressure microinjector apparatus.

2. Remove host embryos from humidified incubator and place in an egg holder underneath the fluorescent stereo dissecting microscope.

1. Open the vitelline membrane using sterile fine forceps and make a small incision $(\sim 0.5-1.0 \mathrm{~mm}$ in length) in the pericardium. Additional manipulation/dissection may be needed depending on target region for injection.

3. Position the microinjector such that the tip of the microinjection needle penetrates the target tissue.

1. Pressure inject cells, and use the fluorescent label to determine that implanted cells are present in desired tissue. For typical injections, apply single pulses less than $0.5 \mathrm{~s}$ in duration ranging from 100-400 hectopascals in pressure.

NOTE: Pulse length and absolute pressure will vary depending on the number of cells to be injected and can be modified to suit individual needs.

2. Retract the microinjector apparatus and remove the egg from the holder after pressure injecting.

4. Add $1 \mathrm{~mL}$ of warm HBSS dropwise onto the embryo, seal eggs using transparent packing tape, and incubate in the humidified incubator at 38 ${ }^{\circ} \mathrm{C}$ for $24 \mathrm{~h}$ post implantation.

\section{Isolation and analysis}

1. Isolate host embryos in RT HBSS using forceps, tenotomy scissors, and microspatula similar to step 4.3 , and fix in $4 \%$ PFA overnight at $4{ }^{\circ} \mathrm{C}$ with gentle rocking.

2. Wash embryos $3 \times 5$ min in HBSS at RT with gentle rocking, and store in HBSS at $4{ }^{\circ} \mathrm{C}$ for further downstream analysis (microscopy, immunohistochemistry, in-situ hybridization, etc.).

\section{Representative Results}

After $24 \mathrm{~h}$ incubation, the heart and surround tissue of host embryos were isolated, photographed (Figure 1A), and processed for immunofluorescent analysis. In this example, donor atrial myocytes were microinjected into the proepicardium of a similar staged host embryo. The host embryo was then stained with the muscle marker (MF20 green) and 4',6-diamidino-2-phenylindole (DAPI; blue). Injected cells (red) are clearly visible (Figure 1B). Possible adjustments to consider if cells are not visible include: donor tissue was over digested (cells would be unable to attach), labeling dye solution was too dilute, cells were over-washed, or multiple cell divisions resulted in loss of the label.

To confirm that the injected cells in this example were myocardial, we optically sectioned this embryo using a confocal microscope (Figure 1CE). The only MF20 positive cells within the proepicardium (PE) are the fluorescent red positive cells that were focally implanted. 


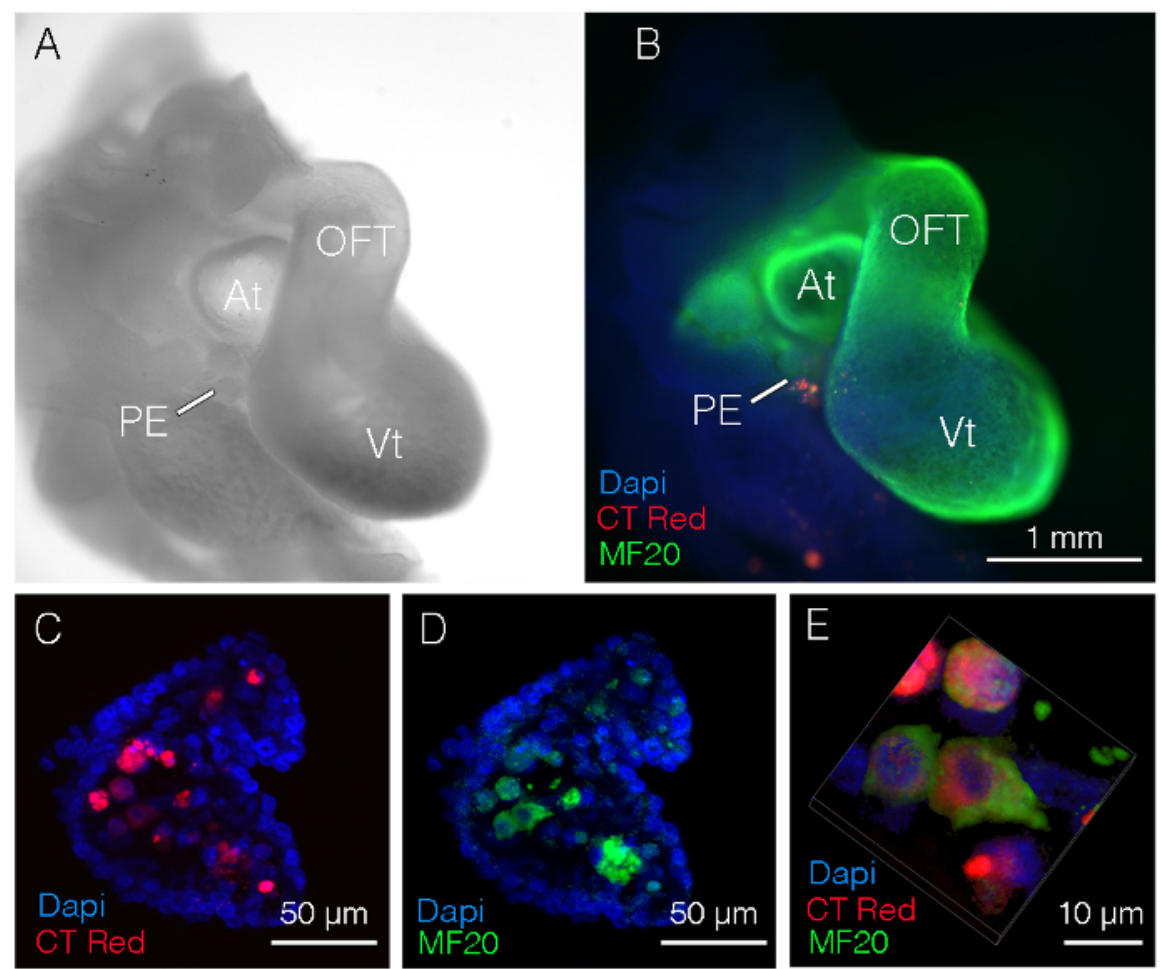

Figure 1: Representative images of embryos isolated 24 hours post injection. (A) Low magnification brightfield image of the trunk region of an E3.5 (HH Stage 19) chick embryo. (B) Merged image showing injected cells (red), cardiomyocytes (green), and DAPI. Cells were isolated from the atria and microinjected into the proepicardium. (C) High magnification confocal imaging showing labeled cells in the core of the proepicardium. (D) High magnification confocal imaging confirming CT Red labeled cells are cardiomyocytes. (E) Three-dimensional (3D) reconstruction of injected cells from panels D and E. At, atria; OFT, outflow tract; PE, proepicardium; Vt, ventricle; MF20, Myosin 4. Please click here to view a larger version of this figure.

\section{Discussion}

The ability to define how microenvironmental conditions impact cardiac cell fate specification and lineage stabilization is fundamental to creating a compressive understanding of congenital heart disease as well as to developing efficient protocols for proper maturation of stem cell or somatic cell reprograming-based cardiomyocytes. The protocol outlined above gives investigators the ability to directly assay cardiac cell development under altered in vivo conditions, allowing for cell autonomous maturation processes to be separated from paracrine/juxtacrine and/ or hemodynamic cues. When combined with high resolution imaging, genetic analysis, and physiological assays, this technique can serve as a powerful complement to existing transgenic models.

Form a technical stand point, the protocol presented here relies on efficient isolation, labeling, and precise implantation of donor heart cells into host embryonic tissues. The use of a microinjection system greatly aids in the targeting of the donor cells and allows for successful implantation without the need for creating a large engraftment site in the host tissue. Some operational skill is required to perform this technique however, as reduced viability can result if the injection needle is not carefully placed in the target tissue (causing rupture of the heart or local vasculature). Care and thought should also be given to the isolation and labeling steps. Over digestion of the donor tissue can lead to poor implantation efficiency, and transient labeling techniques can limit the time window over which donor cells can be tracked (as cell division can dilute the label).

This technique is highly modifiable and can be adapted for a variety of purposes. For example, donor cells from a large range of tissues and stages can be isolated (though optimization of the enzymatic digestion is required) and can similarly be injected into a variety of host tissues across different stages of development. Similarly, the labeling approach can be modified to track cells across different temporal windows, including the use of fluorescent inorganic semiconductor nanocrystals for longer transient labeling and implantation of quail cells or cells from green fluorescent protein transgenic (GFP+) donor embryos ${ }^{11}$ for permeant labeling.

While we currently use this technique for avian implantation studies, we feel that it could be used for a large range of chimeric studies in the future. For example, genetically altered cardiac cells from transgenic organisms could be isolated and microinjected into the avian heart using a very similar protocol. Furthermore, cells differentiated into cardiomyocytes from stems cells or via somatic cell reprograming approaches could be microinjected into the embryonic heart to evaluate their integration into the tissue and/or maturation under in vivo biomechanical conditions.

Disclosures

The authors have nothing to disclose. 


\section{Acknowledgments}

This work was supported by grant R00HL122360 from the National Institutes of Health, National Heart, Lung, and Blood Institute (NHLBI).

\section{References}

1. Guo, Y. X., Pu, W. T. Genetic Mosaics for Greater Precision in Cardiovascular Research. Circulation Research. 123, 27-29 (2018).

2. Guo, Y. X. et al. Analysis of Cardiac Myocyte Maturation Using CASAAV, a Platform for Rapid Dissection of Cardiac Myocyte Gene Function In Vivo. Circulation Research. 120 (12), 1874-1888 (2017).

3. Rugh, R. Experimental embryology; techniques and procedures. 3rd edition, Burgess Pub. Co., (1962).

4. Slack, J. M. W. Essential developmental biology. 3rd edition, Wiley, (2013).

5. Reinecke, H., Zhang, M., Bartosek, T., Murray, C. E. Survival, integration, and differentation of cardiomyocyte grafts: a study in normal and injured rat hearts. Circulation. 100, 193-202 (1999).

6. Rojas, S. V. et al. Transplantation of purified iPSC-derived cardiomyocytes in myocardial infarction. PLOS ONE. 12, e0173222 (2017).

7. Zhang, M. et al. Cardiomyocyte Grafting for Cardiac Repair: Graft Cell Death and Anti-Death Strategies. Journal of Molecular and Cellular Cardiology. 33 (5), 907-921 (2001).

8. Bressan, M., Liu, G., Mikawa, T. Early mesodermal cues assign avian cardiac pacemaker fate potential in a tertiary heart field. Science. $\mathbf{3 4 0}$ (6133), 744-748 (2013).

9. Bressan, M. et al. Reciprocal myocardial-endocardial interactions pattern the delay in atrioventricular junction conduction. Development. 141 (21), 4149-4157 (2014).

10. Hamburger, V., Hamilton, H. L. A series of normal stages in the development of the chick embryo. Journal of Morphology. 88 (1), $49-92$ (1951). 